ESAIM: PROCEEDINGS, December 2008, Vol. 25, p. 1-18

E. Cancès, S. Faure, B. Graille, Editors

\title{
SOLUTIONS FORTES DES ÉQUATIONS DE NAVIER-STOKES AVEC CONDITIONS DISSIPATIVES NATURELLES *
}

\author{
C. Grandmont ${ }^{1}$ And A. Sounlah ${ }^{2}$
}

\begin{abstract}
We are interested in the modelling of the air flow in the respiratory tract. We propose a decomposition of the respiratory tree into two stages: a proximal part, the trachea and the first generations of bronchial tubes (around the fifth or the sixth one) and a distal part, corresponding to the complex geometrical part of the bronchial tree. In the upper part, we assume that the flow is governed by the Navier-Stokes equations. The lower part is a network of tubes of low diameters and we suppose that, in each tube, the flow is described by the Poiseuille law and thus regulated by the pressure drop between the inlets and the outlets. Then each network can be condensed and replaced by a non standard boundary condition for the Navier-Stokes system. Such boundary condition is also used for heamodynamics modelling, and in this paper we provide a well posedness analysis of this multiscale model by proving the existence and uniqueness of a solution locally in time for any data, and the existence and uniqueness of solutions globally in time for small data. The continuous dependance of the solution with respect to the data is also proved.
\end{abstract}

Résumé. On s'intéresse à la modélisation multiéchelle pour la description de flux biologiques et en particulier de l'air dans les voies aériennes supérieures. Pour cela on décompose en deux niveaux l'arbre bronchique. Dans la partie proximale (les six premières générations), on suppose que l'écoulement est régi par les équations de Navier-Stokes. Dans la partie distale (de la génération sept à la génération dix sept), on suppose que les flux à travers chacune des bronches sont régis par la loi de Poiseuille. Dans chacun des sous arbres de la partie distale les écoulements sont alors caractérisés par une résistance et peuvent s'écrire sous forme d'une condition aux limites non standard pour les équations de NavierStokes. Pour ce modèle multiéchelle, utilisé également dans le cadre des écoulements sanguins, nous démontrons l'existence d'une unique solution locale en temps ainsi qu'un résultat d'existence et d'unicité globale en temps pour données petites. La dépendance continue de la solution par rapport aux données est également démontrée.

\section{INTRODUCTION}

La modélisation mathématique est de plus en plus sollicitée par le monde de la santé. De tels modèles sont utilisés pour une meilleure compréhension des fonctions physiologiques et peuvent aider à développer de nouvelles méthodes de diagnostic et de nouvelles techniques thérapeutiques. La modélisation du système respiratoire s'inscrit dans ce contexte, où par exemple, la mise en place d'un poumon numérique aiderait certainement à

\footnotetext{
* Ce travail a été financé par le projet ACINIM Lepoumonvousdisje et l'Ambassade de France en Tunisie.

1 INRIA, Projet REO Rocquencourt, BP 10578153 Le Chesnay Cedex, France

2 Laboratoire de mathématiques , Université Paris-Sud Bâtiment 425, bureau 130, 91405 Orsay Cedex, France
} 
mieux comprendre certaines pathologies et guiderait mieux les stratégies curatives. Cet article est motivé par la recherche d'un modèle permettant de reproduire l'écoulement de l'air dans la totalité de l'arbre bronchique humain. Ce projet se heurte à deux principales difficultés :

(1) La complexité de la géométrie de l'arbre bronchique et la difficulté de résoudre des équations aux dérivées partielles dans une telle géométrie (l'arbre bonchique contient typiquement $2^{23}$ générations, soit environ 8 millions de sorties [23]).

(2) La définition de conditions aux limites pertinentes, adaptées au phénomène ventilatoire, sur les multiples sorties du domaine.

L'approche que nous proposons est similaire aux approches multiéchelles pour la modélisation de l'écoulement du sang dans le réseau artériel ( [18], [20], [22]) et consiste à distinguer dans l'arbre deux zones distinctes : une zone proximale qui correspond à la trachée et aux premières bronches où on suppose que l'écoulement est gouverné par les équations de Navier-Stokes et où des simulations directes peuvent avoir lieu, et une partie distale, correspondant à la partie géométriquement complexe et non accessible, à l'heure actuelle, par les techniques standard d'imagerie médicale. L'utilisation des équations de Navier-Stokes pour modéliser l'écoulement de l'air dans la partie proximale est pertinente, au moins, en ce qui concerne la respiration calme ( [7], [8], [9]). Il est à noter que, dans ce type de régime oscillant, les instabilités ne semblent pas avoir le temps de se développer. La partie distale, localisée en aval de la partie proximale de l'arbre, ne fera pas partie du domaine de calcul et est destinée à être condensée. Ici on suppose que cette zone est composée d'un réseau de tubes de petits diamètres dans lesquels un écoulement de Stokes linéaire, visqueux, incompressible a lieu, régulé par les différences de pression entre entrées et sorties. Ainsi la loi de Poiseuille est satisfaite dans chacun des tubes et dans chacun des sous arbres, l'écoulement est caractérisé par sa "résistance".

Plus précisément la géométrie que l'on considère est schématiquement représentée dans la figure 1.

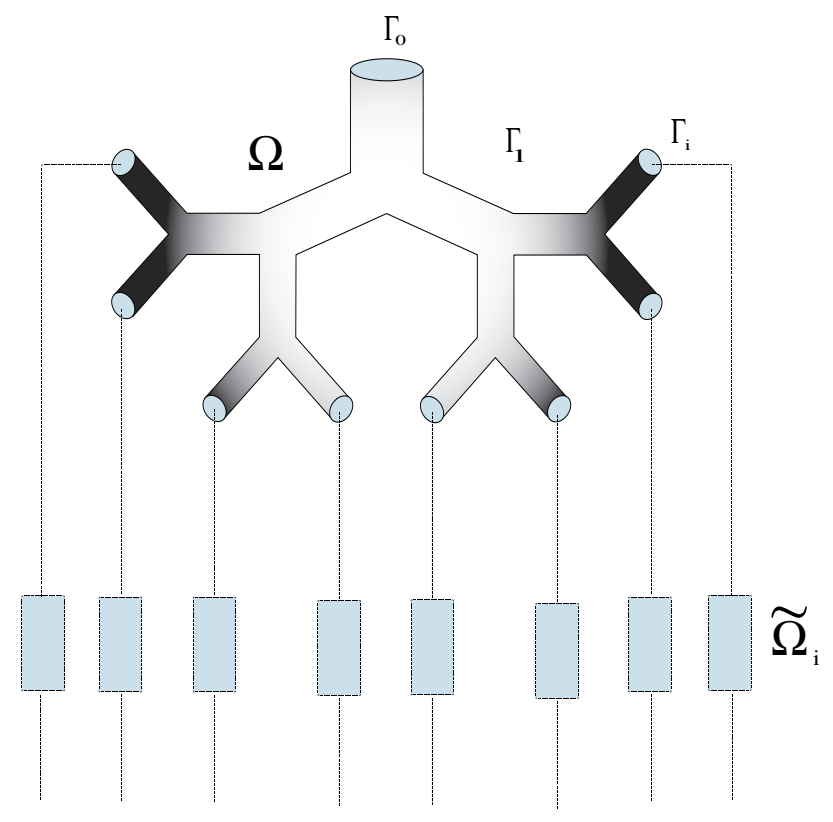

Figure 1. Le domaine

Le domaine $\Omega$ correspond aux premières bronches. La frontière $\partial \Omega$ du domaine est la réunion de la paroi latérale $\Gamma_{l}$, d'une section d'entrée unique qui correspond au nez, le larynx et l'entrée de la trachée, qu'on 
désignera par $\Gamma_{0}$ et de multiples sorties $\Gamma_{i}, i=1, \ldots, N$. Notons que $\Gamma_{0}$ n'est une vraie entrée que pendant la phase inspiratoire, mais par souci de simplification, on utilisera toujours cette terminologie. On suppose que la pression est uniforme au niveau de chacune des sorties $\Gamma_{i}$ (on note $\Pi_{i}$ les valeurs correspondantes), et on désigne par $P_{0}$ la pression atmosphérique au niveau de $\Gamma_{0}$. Si on prescrit des conditions naturelles, non dissipative, associée à la pression en entrée $P_{0}$ et aux pressions $\Pi_{i}$ au niveau de chaque sortie, le système qu'on considère au niveau du domaine $\Omega$ s'écrit

$$
\left\{\begin{aligned}
\rho \frac{\partial \mathbf{u}}{\partial t}+\rho(\mathbf{u} \cdot \nabla) \mathbf{u}-\mu \Delta \mathbf{u}+\nabla \mathbf{p} & =0 & & \operatorname{dans} \Omega, \\
\nabla \cdot \mathbf{u} & =0 & & \operatorname{dans} \Omega, \\
\mathbf{u} & =0 & & \text { sur } \Gamma_{l}, \\
\mu \nabla \mathbf{u} \cdot \mathbf{n}-\mathrm{p} \mathbf{n} & =-P_{0} \mathbf{n} & & \text { sur } \Gamma_{0}, \\
\mu \nabla \mathbf{u} \cdot \mathbf{n}-\mathrm{p} \mathbf{n} & =-\Pi_{i} \mathbf{n} & & \text { sur } \Gamma_{i}, i=1, \ldots, N .
\end{aligned}\right.
$$

La partie distale de l'arbre correspond à la réunion des domaines $\tilde{\Omega}_{i}, i=1, \ldots, N$. Il est à noter que chaque sous domaine $\tilde{\Omega}_{i}$ a à son tour une structure géométrique dichotomique ramifiée. Dans chacun des sousdomaines $\tilde{\Omega}_{i}$, et grâce aux petits diamètres des sections des tubes, on suppose que l'écoulement dans les petites bronches est gouverné par la loi de Poiseuille. Cette loi est analogue à la relation électrique dans un réseau dans lequel le débit s'apparente à l'intensité tandis que la pression joue le rôle du potentiel électrique. Ainsi, dans le cas de l'arbre bronchique humain, on peut condenser la partie distale et représenter, sans perte de généralité, chaque sous domaine tronqué $\tilde{\Omega}_{i}$ comme un tube unique où l'écoulement est gouverné par une loi de Poiseuille généralisée [12], [17]. Par conséquent, il existe une constante $R_{i}$ ("résistance" de chaque sous arbre $i$ ) qui dépend de la géométrie, telle que

$$
\Pi_{i}-P_{i}=R_{i} \int_{\Gamma_{i}} \mathbf{u} \cdot \mathbf{n},
$$

où les pressions $P_{i}$ correspondent à la pression au niveau des alvéoles associées à la ième bronche de l'arbre. En éliminant la pression de sortie $\Pi_{i}$ grâce à la relation précédente, on obtient les équations da Navier-Stokes avec des conditions aux limites "dissipatives" non standard

$$
\left\{\begin{aligned}
\rho \frac{\partial \mathbf{u}}{\partial t}+\rho(\mathbf{u} \cdot \nabla) \mathbf{u}-\mu \Delta \mathbf{u}+\nabla \mathbf{p} & =0 & & \operatorname{dans} \Omega, \\
\nabla \cdot \mathbf{u} & =0 & & \operatorname{dans} \Omega, \\
\mathbf{u} & =0 & & \text { sur } \Gamma_{l} \\
\mu \nabla \mathbf{u} \cdot \mathbf{n}-\mathbf{p} \mathbf{n} & =-P_{0} \mathbf{n} & & \text { sur } \Gamma_{0}, \\
\mu \nabla \mathbf{u} \cdot \mathbf{n}-\mathbf{p} \mathbf{n} & =-P_{i} \mathbf{n}-R_{i}\left(\int_{\Gamma_{i}} \mathbf{u} \cdot \mathbf{n}\right) \mathbf{n} & & \text { sur } \Gamma_{i}, i=1, \ldots, N .
\end{aligned}\right.
$$

Dans ce qui suit nous désignerons ces conditions aux limites comme étant des conditions naturelles dissipatives.

L'avantage de cette approche consiste donc à restreindre le domaine de calcul $(\Omega$ couvre juste les cinq ou six premières générations de bronches au lieu des 24 générations de l'arbre complet), tout en tenant compte de l'écoulement dans le reste de l'arbre. Dans [2] ce modèle est couplé à un troisième compartiment de manière à prendre en compte les acini ainsi que le mouvement du diaphragme. Dans [13] une contrainte supplémentaire est rajoutée au modèle de manière à ce que la trace des vitesses sur les sections d'entrées et de sorties soit réduite à un seul degré de liberté. Enfin, notons que ce type d'approche multiéchelle est également utilisée pour la simulation des écoulements sanguins [18], [20], [22].

Ici nous nous intéressons uniquement à l'existence de solutions de (3). Dans [20] l'existence de solutions fortes est démontrée pour des conditions aux limites du type $\mu \nabla \mathbf{u} \cdot \mathbf{n}-\mathbf{p} \mathbf{n}=-P_{i} \mathbf{n}-R_{i}(\mathbf{u} \cdot \mathbf{n}) \mathbf{n}$, sous une condition de petitesse des résistances $R_{i}$. Nous allons reprendre les mêmes techniques de démonstration mais en nous affranchissant de ce type de condition. 
Cet article s'organise comme suit : dans un premier temps, on étudie le problème dans le cas des équations de Stokes stationnaires ainsi que les propriétés relatives à l'opérateur de Stokes avec des conditions aux limites naturelles dissipatives. Ensuite, on s'intéresse au cas des équations de Navier-Stokes. Le principe de démonstration est le même que celui développé dans [15] ou [20]. Le problème est approché par la méthode de Galerkin. Contrairement à [15] ou [20], où les fonctions de la base de Galerkin sont les fonctions propres de l'opérateur de Stokes associé à des conditions aux limites mixtes Dirichlet/Neumann, ici ce sont les fonctions propres associées à l'opérateur de Stokes mais avec des conditions aux limites mixtes Dirichlet/naturelles dissipatives. Grâce à ce choix, nous obtenons des estimations uniformes sur la solution approchée sans condition sur la taille des résistances, la principale difficulté, à cette étape, étant d'estimer le terme non linéaire provenant du flux d'énergie cinétique. Enfin, ces estimations nous permettent de passer à la limite dans les équations et de prouver qu'il existe un intervalle de temps sur lequel le problème admet une solution. De plus, on montre que le problème admet une solution globale en temps si les données sont suffisamment petites. La dépendance continue par rapport aux données et l'unicité de la solution sont également démontrées.

\section{Le PRoblème de Stokes Stationnaire}

Dans cette section, on s'intéresse à l'étude du problème de Stokes stationnaire avec conditions aux limites dissipatives, où il s'agit de trouver $\mathbf{u} \in H^{1}(\Omega)$ et $\mathrm{p} \in L^{2}(\Omega)$ solutions de

$$
\left\{\begin{aligned}
-\mu \Delta \mathbf{u}+\nabla \mathbf{p} & =\mathbf{f} & & \operatorname{dans} \Omega, \\
\nabla \cdot \mathbf{u} & =0 & & \operatorname{dans} \Omega, \\
\mathbf{u} & =0 & & \text { sur } \Gamma_{l}, \\
\mu \nabla \mathbf{u} \cdot \mathbf{n}-\mathbf{p} \mathbf{n} & =-P_{0} \mathbf{n} & & \text { sur } \Gamma_{0}, \\
\mu \nabla \mathbf{u} \cdot \mathbf{n}-\mathbf{p} \mathbf{n} & =-P_{i} \mathbf{n}-R_{i}\left(\int_{\Gamma_{i}} \mathbf{u} \cdot \mathbf{n}\right) \mathbf{n} & & \text { sur } \Gamma_{i} \quad i=1, \ldots, N,
\end{aligned}\right.
$$

avec les pressions $P_{i}, i=0, \ldots, N$, les résistances $R_{i}>0, i=1, \ldots, N$ et la force volumique $\mathbf{f} \in L^{2}(\Omega)$ données.

On se propose d'exprimer (4) en une formulation variationnelle équivalente. Vue la condition aux limites de non glissement sur $\Gamma_{l}$, on choisit l'espace suivant pour la vitesse

$$
H_{0, l}^{1}(\Omega)=\left\{\mathbf{v} \in H^{1}(\Omega)^{d}, \mathbf{v} / \Gamma_{l}=0\right\}
$$

où $d=2,3$. Sur cet espace la semi norme $H^{1}$ est équivalente à la norme $H^{1}$ (puisque mes $\left(\Gamma_{l}\right) \neq 0$ ). Les conditions aux limites en entrée sur $\Gamma_{0}$ et sur les multiples sorties $\Gamma_{i}, i=1, \ldots, N$, déterminent la pression de référence; la pression n'est donc pas définie à une constante additive près et l'espace en pression est

$$
M=L^{2}(\Omega)
$$

et d'une manière usuelle on définit l'espace des vitesse à divergence nulle

$$
V=\left\{\mathbf{v} \in H_{0, l}^{1}(\Omega), \nabla \cdot \mathbf{v}=0\right\}
$$

Noter que $V$ est un espace de Hilbert comme fermé de $H_{0, l}^{1}(\Omega)$, qui l'est également comme fermé de $H^{1}(\Omega)$. En faisant le produit scalaire de la première équation de $(4)$ dans $L^{2}(\Omega)$ avec une fonction test $\tilde{\mathbf{u}} \in H_{0, l}^{1}(\Omega)$, et en appliquant la formule de Green, on obtient

$$
\mu \int_{\Omega} \nabla \mathbf{u}: \nabla \tilde{\mathbf{u}}-\int_{\Omega} \mathrm{p} \nabla \cdot \tilde{\mathbf{u}}+\int_{\partial \Omega}(-\mu \nabla \mathbf{u} \cdot \mathbf{n}+\mathrm{p} \mathbf{n}) \cdot \tilde{\mathbf{u}}=\int_{\Omega} \mathbf{f} \cdot \tilde{\mathbf{u}}, \forall \tilde{\mathbf{u}} \in H_{0, l}^{1}(\Omega) .
$$


En prenant en compte les conditions aux limites apparaissant dans (4), la formulation variationnelle du problème avec conditions de sorties naturelles dissipatives s'écrit : pour $\mu,\left(P_{i}\right)_{i=0, N},\left(R_{i}\right)_{i=1, N}$ et $\mathbf{f} \in L^{2}(\Omega)$ donnés, trouver $\mathbf{u} \in H_{0, l}^{1}(\Omega)$ et $\mathrm{p} \in M$ solutions de

$$
\left\{\begin{array}{l}
\mu \int_{\Omega} \nabla \mathbf{u}: \nabla \tilde{\mathbf{u}}+\sum_{i=1}^{N} R_{i}\left(\int_{\Gamma_{i}} \mathbf{u} \cdot \mathbf{n}\right)\left(\int_{\Gamma_{i}} \tilde{\mathbf{u}} \cdot \mathbf{n}\right)-\int_{\Omega} \mathrm{p} \nabla \cdot \tilde{\mathbf{u}}= \\
-P_{0} \int_{\Gamma_{0}} \tilde{\mathbf{u}} \cdot \mathbf{n}-\sum_{i=1}^{N} P_{i} \int_{\Gamma_{i}} \tilde{\mathbf{u}} \cdot \mathbf{n}+\int_{\Omega} \mathbf{f} \cdot \tilde{\mathbf{u}}, \forall \tilde{\mathbf{u}} \in H_{0, l}^{1}(\Omega), \\
\int_{\Omega} \mathrm{q} \nabla \cdot \mathbf{u}=0, \forall \mathrm{q} \in M .
\end{array}\right.
$$

On vient de montrer que toute solution $(\mathbf{u}, \mathrm{p}) \in H_{0, l}^{1}(\Omega) \times M$ de $(4)$ est aussi solution de la formulation variationnelle (5). Réciproquement, si (u, p) est solution du problème variationnel (5), en choisissant $\tilde{\mathbf{u}} \in \mathcal{D}(\Omega)$, on obtient la première équation de (4) au sens des distributions. La deuxième équation de (5) est équivalente à contrainte d'incompressibilité. Bien sûr, le fait que u appartienne à $H_{0, l}^{1}(\Omega)$, implique la condition de nonglissement sur la paroi $\Gamma_{l}$. Pour récupérer les conditions aux limites sur les $\Gamma_{i}, i=0, \ldots, N$, on procède de manière standard et on multiplie la première équation de $(4)$, qui est vraie dans $L^{2}(\Omega)\left(\operatorname{car} \mathbf{f} \in L^{2}(\Omega)\right)$, par $\tilde{\mathbf{u}} \in H_{0, l}^{1}(\Omega)$ et l'on intègre par parties. En retranchant la formulation faible, il ne reste plus que des contributions au bord et on en déduit les conditions aux limites sur $\Gamma_{i}$ qui ont un sens dans $\left(H_{00}^{\frac{1}{2}}\left(\Gamma_{i}\right)\right)^{\prime}$. Ainsi, on a établit la proposition suivante

Proposition 1.1. Les problèmes (4) et (5) sont équivalents.

\subsection{Existence et unicité}

Le problème (5) est de type mixte. En effet, si on introduit les notations suivantes

$$
\begin{aligned}
a(\mathbf{u}, \tilde{\mathbf{u}}) & =\mu \int_{\Omega} \nabla \mathbf{u}: \nabla \tilde{\mathbf{u}}+\sum_{i=1}^{N} R_{i}\left(\int_{\Gamma_{i}} \mathbf{u} \cdot \mathbf{n}\right)\left(\int_{\Gamma_{i}} \tilde{\mathbf{u}} \cdot \mathbf{n}\right), \\
b(\tilde{\mathbf{u}}, \mathrm{q}) & =-\int_{\Omega} \mathrm{q} \nabla \cdot \tilde{\mathbf{u}}, \\
l(\tilde{\mathbf{u}}) & =-P_{0} \int_{\Gamma_{0}} \tilde{\mathbf{u}} \cdot \mathbf{n}-\sum_{i=1}^{N} P_{i} \int_{\Gamma_{i}} \tilde{\mathbf{u}} \cdot \mathbf{n}+\int_{\Omega} \mathbf{f} \cdot \tilde{\mathbf{u}},
\end{aligned}
$$

le problème (5) s'écrit sous la forme : $H_{0, l}^{1}(\Omega)$ et $M$ étant deux espaces de Hilbert, $a$ et $b$ deux formes bilinéaires respectivement sur $H_{0, l}^{1}(\Omega) \times H_{0, l}^{1}(\Omega)$ et $H_{0, l}^{1}(\Omega) \times M, l$ une forme linéaire sur $H_{0, l}^{1}(\Omega)$, trouver $\mathbf{u} \in H_{0, l}^{1}(\Omega)$ et $\mathrm{p} \in M$ solutions de

$$
\left\{\begin{aligned}
a(\mathbf{u}, \tilde{\mathbf{u}})+b(\tilde{\mathbf{u}}, \mathrm{p}) & =l(\tilde{\mathbf{u}}), \forall \tilde{\mathbf{u}} \in H_{0, l}^{1}(\Omega), \\
b(\mathbf{u}, \mathrm{q}) & =0, \forall \mathrm{q} \in M .
\end{aligned}\right.
$$

Ainsi, le fait que (5) soit bien posé, est la conséquence des propriétés suivantes : la coercivité de la forme bilinéaire $a(\cdot, \cdot)$ sur $V$, la condition inf-sup sur $H_{0, l}^{1}(\Omega) \times M$ pour la forme bilinéaire $b(\cdot, \cdot)$ et la continuité de la forme linéaire $l(\cdot)$. 
(1) Coercivité de $a$ sur $V$ :

L'ellipticité de $a$ est immédiate car les $R_{i}$ sont positives et que $\operatorname{mes}\left(\Gamma_{l}\right) \neq 0$

$$
\begin{aligned}
a(\mathbf{u}, \mathbf{u}) & =\mu\|\nabla \mathbf{u}\|_{L^{2}(\Omega)}^{2}+\sum_{i=1}^{N} R_{i}\left(\int_{\Gamma_{i}} \mathbf{u} \cdot \mathbf{n}\right)^{2} \\
& \geq \mu\|\nabla \mathbf{u}\|_{L^{2}(\Omega)}^{2}, \forall \mathbf{u} \in H_{0, l}^{1}(\Omega) .
\end{aligned}
$$

La forme bilinéaire $a(\cdot, \cdot)$ est coercive sur $H_{0, l}^{1}(\Omega)$, elle l'est donc aussi sur $V$.

(2) Condition inf-sup :

La condition inf-sup est établie par la proposition suivante, dont la preuve sera détaillée plus loin

Proposition 1.2. Il existe une constante $\beta>0$ telle que

$$
\forall q \in M, \sup _{\mathbf{v} \in H_{0, l}^{1}(\Omega)} \frac{1}{\|\nabla \mathbf{v}\|_{L^{2}(\Omega)}} \int_{\Omega} q \nabla \cdot \mathbf{v} \geq \beta\|q\|_{L^{2}(\Omega)} .
$$

(3) Continuité de $l(\cdot)$ :

On a

$$
|l(\tilde{\mathbf{u}})| \leq C\left(\max _{i=0, \ldots, N}\left|P_{i}\right|+\|\mathbf{f}\|_{L^{2}(\Omega)}\right)\|\tilde{\mathbf{u}}\|_{H^{1}(\Omega)}, \forall \tilde{\mathbf{u}} \in H^{1}(\Omega) .
$$

Finalement, grâce à la théorie de Babuška-Brezzi ( [1], [5]) sur les problèmes de type point-selle, on aboutit à la proposition suivante

Proposition 1.3. Le problème (5) est bien posé et admet une unique solution (u,p) dans $V \times M$.

Nous détaillons maintenant la preuve de la condition inf-sup énoncée à la proposition 1.2 : Démonstration. Soit $q \in M$. On décompose $q$ sous la forme $q=q^{*}+\bar{q}$ où $\bar{q}=\frac{1}{|\Omega|} \int_{\Omega} q$. Ainsi

$$
q^{*} \in L_{0}^{2}(\Omega)=\left\{q \in L^{2}(\Omega), \int_{\Omega} q=0\right\}
$$

et de plus on a

$$
\int q^{*} \bar{q}=0 \text { et donc }\|q\|_{L^{2}(\Omega)}^{2}=\left\|q^{*}\right\|_{L^{2}(\Omega)}^{2}+\|\bar{q}\|_{L^{2}(\Omega)}^{2} .
$$

Comme $q^{*} \in L_{0}^{2}(\Omega)$, il existe $\mathbf{v}^{*} \in H_{0}^{1}(\Omega)$ tel que

$$
\nabla \cdot \mathbf{v}^{*}=q^{*} \text { et }\left\|\nabla \mathbf{v}^{*}\right\|_{L^{2}(\Omega)} \leq \frac{1}{\beta^{*}}\left\|q^{*}\right\|_{L^{2}(\Omega)},
$$

où la constante $\beta^{*}$ dépend seulement du domaine $\Omega$ (voir [11]).

La technique utilisée dans [4] et [10] consiste à associer à $\bar{q}$ un élément $\overline{\mathbf{v}}$ adéquat et à exprimer $\mathbf{v}$ comme une combinaison linéaire adéquate de $\mathbf{v}^{*}$ et $\overline{\mathbf{v}}, \mathbf{v}=\gamma \mathbf{v}^{*}+\overline{\mathbf{v}}$. Ainsi

$$
\begin{aligned}
\int_{\Omega} q \nabla \cdot \mathbf{v} & =\int_{\Omega}\left(q^{*}+\bar{q}\right)\left(\gamma \nabla \cdot \mathbf{v}^{*}+\nabla \cdot \overline{\mathbf{v}}\right) \\
& =\int_{\Omega} \gamma q^{*} \nabla \cdot \mathbf{v}^{*}+\int_{\Omega} q^{*} \nabla \cdot \overline{\mathbf{v}}+\int_{\Omega} \gamma \bar{q} \nabla \cdot \mathbf{v}^{*}+\int_{\Omega} \bar{q} \nabla \cdot \overline{\mathbf{v}} .
\end{aligned}
$$

Comme $\nabla \cdot \mathbf{v}^{*}=q^{*}$, on a

$$
\int_{\Omega} \gamma q^{*} \nabla \cdot \mathbf{v}^{*}=\gamma\left\|q^{*}\right\|_{L^{2}(\Omega)}^{2} \text { et } \int_{\Omega} \gamma \bar{q} \nabla \cdot \mathbf{v}^{*}=0
$$


En ce qui concerne le terme $\int_{\Omega} \bar{q} \nabla \cdot \overline{\mathbf{v}}$, on choisit $\overline{\mathbf{v}}$ tel que

$$
\int_{\Omega} \bar{q} \nabla \cdot \overline{\mathbf{v}}=\|\bar{q}\|_{L^{2}(\Omega)}^{2}
$$

En effet on définit $\overline{\mathbf{v}}$ par

$$
\overline{\mathbf{v}}=|\Omega| \bar{q} \rho \mathbf{n}_{0},
$$

où $\mathbf{n}_{0}$ est la normale sortante à la section $\Gamma_{0}$ (ici on suppose pour simplifier que $\mathbf{n}_{0}=(0,0,1)$ ), $\rho$ une fonction régulière, qui s'annule sur $\partial \Omega \backslash \Gamma_{0}$ et satisfait

$$
\int_{\Gamma_{0}} \rho=1
$$

Ainsi

$$
\int_{\Omega} \bar{q} \nabla \cdot \overline{\mathbf{v}}=|\Omega| \bar{q}^{2}\left(\int_{\Gamma_{0}} \rho\right)=\|\bar{q}\|_{L^{2}(\Omega)}^{2}
$$

Maintenant on a

$$
\int_{\Omega} q \nabla \cdot \mathbf{v}=\gamma\left\|q^{*}\right\|_{L^{2}(\Omega)}^{2}+\|\bar{q}\|_{L^{2}(\Omega)}^{2}+\int_{\Omega} q^{*} \nabla \cdot \overline{\mathbf{v}}
$$

et il reste à minorer le terme croisé $\int_{\Omega} q^{*} \nabla \cdot \overline{\mathbf{v}}$. Par la définition de $\overline{\mathbf{v}}$ et l'inégalité de Cauchy-Schwartz, on a

$$
\begin{aligned}
\int_{\Omega} q^{*} \nabla \cdot \overline{\mathbf{v}} & =|\Omega| \int_{\Omega} q^{*} \nabla \cdot\left(\bar{q} \rho \mathbf{n}_{0}\right) \\
& =|\Omega| \bar{q} \int_{\Omega} q^{*} \nabla \rho \cdot \mathbf{n}_{0} \\
& \geq-|\Omega|^{\frac{1}{2}}\|\nabla \rho\|_{L^{2}(\Omega)}\left\|q^{*}\right\|_{L^{2}(\Omega)}\|\bar{q}\|_{L^{2}(\Omega)}
\end{aligned}
$$

Finalement, en choisissant $\gamma=|\Omega|\|\nabla \rho\|_{L^{2}(\Omega)}^{2}$ et en remplaçant dans (7), on obtient l'estimation

$$
\int_{\Omega} q \nabla \cdot \mathbf{v} \geq \gamma\left\|q^{*}\right\|_{L^{2}(\Omega)}^{2}+\|\bar{q}\|_{L^{2}(\Omega)}^{2}-|\Omega|^{\frac{1}{2}}\|\nabla \rho\|_{L^{2}(\Omega)}\left\|q^{*}\right\|_{L^{2}(\Omega)}\|\bar{q}\|_{L^{2}(\Omega)}
$$

L'inégalité $a^{2}+b^{2}-a b \geq \frac{1}{2}\left(a^{2}+b^{2}\right)$ nous permet de déduire

$$
\begin{aligned}
\int_{\Omega} q \nabla \cdot \mathbf{v} & \geq \frac{1}{2}\left(|\Omega|\|\nabla \rho\|_{L^{2}(\Omega)}^{2}\left\|q^{*}\right\|_{L^{2}(\Omega)}^{2}+\|\bar{q}\|_{L^{2}(\Omega)}^{2}\right) \\
& \geq C_{1}\|q\|_{L^{2}(\Omega)}^{2} .
\end{aligned}
$$
où $C_{1}=\frac{1}{2} \min \left(|\Omega|\|\nabla \rho\|_{L^{2}(\Omega)}^{2}, 1\right)$. Par ailleurs, grâce au choix de $\gamma$, à la propriété (6) et à l'expression de $\overline{\mathbf{v}}$,
on a

$$
\begin{aligned}
\|\nabla \mathbf{v}\|_{L^{2}(\Omega)} & \leq|\gamma|\left\|\nabla \mathbf{v}^{*}\right\|_{L^{2}(\Omega)}+\|\nabla \overline{\mathbf{v}}\|_{L^{2}(\Omega)} \\
& \leq|\Omega|\|\nabla \rho\|_{L^{2}(\Omega)}\left(\frac{\|\nabla \rho\|}{\beta^{*}}\left\|q^{*}\right\|+\frac{1}{|\Omega|^{1 / 2}}\|\bar{q}\|\right) \\
& \leq C_{2}\|q\|_{L^{2}(\Omega)},
\end{aligned}
$$


où $C_{2}=|\Omega|\|\nabla \rho\|_{L^{2}(\Omega)}\left(\left(\frac{\|\nabla \rho\|_{L^{2}(\Omega)}}{\beta^{*}}\right)^{2}+\frac{1}{|\Omega|}\right)^{1 / 2}$.

Grâce aux estimations (8) et (9), on obtient la condition inf-sup avec $\beta=\frac{C_{1}}{C_{2}}$.

\subsection{Etude de l'opérateur de Stokes modifié}

Pour prouver l'existence de solutions pour le système des équations de Navier-Stokes, il est usuel de construire la solution comme limite d'approximations de Galerkin où la base de Galerkin est constituée de vecteurs propres de l'opérateur de Stokes. On peut se référer par exemple à [6], [14], [19], [21], pour le cas de conditions aux limites de Dirichlet sur toute la frontière du domaine, et à [15] pour le cas de conditions aux limites mixtes, où une condition de Dirichlet est imposée sur une partie de la frontière du domaine tandis que des conditions d'entrée et de sorties libres sont imposées sur le reste de la frontière. Ici au lieu de considérer les fonctions propres de l'opérateur de Stokes avec comme conditions aux limites des conditions de Dirichlet ou de Neumann, nous allons considérer les fonctions propres de l'opérateur Stokes avec des conditions aux limites mixtes type Dirichlet et naturelles dissipatives. Ce qui revient à étudier l'opérateur associé à la forme bilinéaire sur $V$, qui prend en compte les termes dissipatifs de notre modèle, soit

$$
a(\mathbf{u}, \tilde{\mathbf{u}})=\int_{\Omega} \nabla \mathbf{u}: \nabla \tilde{\mathbf{u}}+\sum_{i=1}^{N} R_{i}\left(\int_{\Gamma_{i}} \mathbf{u} \cdot \mathbf{n}\right)\left(\int_{\Gamma_{i}} \tilde{\mathbf{u}} \cdot \mathbf{n}\right) .
$$

\subsubsection{Définition et propriétés de l'opérateur}

Rappelons que $V$ est défini par

$$
V=\left\{\mathbf{u} \in H_{0, l}^{1}(\Omega), \nabla \cdot \mathbf{u}=0\right\},
$$

et on considère également $H$ le complété de $V$ par rapport à la norme $L^{2}(\Omega)$. L'espace $V$ est dense dans $H$ et l'injection de $V$ dans $H$ est continue.

Rappelons également qu'on considère la forme bilinéaire

$$
\begin{gathered}
a: V \times V \rightarrow \mathbb{R} \text { telle que } \\
a(\mathbf{u}, \tilde{\mathbf{u}})=\mu \int_{\Omega} \nabla \mathbf{u}: \nabla \tilde{\mathbf{u}}+\sum_{i=1}^{N} R_{i}\left(\int_{\Gamma_{i}} \mathbf{u} \cdot \mathbf{n}\right)\left(\int_{\Gamma_{i}} \tilde{\mathbf{u}} \cdot \mathbf{n}\right),
\end{gathered}
$$

qui est continue et coercive.

Le triplet $\{V, H, a\}$ définit un opérateur $A: D(A) \rightarrow H$ avec

$$
D(A)=\left\{\mathbf{u} \in V / \text { il existe } C \geq 0 \text { telle que }|a(\mathbf{u}, \tilde{\mathbf{u}})| \leq C\|\tilde{\mathbf{u}}\|_{H} \quad \forall \tilde{\mathbf{u}} \in V\right\} .
$$

C'est à dire que $D(A)$ est l'ensemble des $\mathbf{u} \in V$ tels que la forme linéaire $\mathbf{v} \mapsto a(\mathbf{u}, \mathbf{v})$ définie sur $V$, est continue pour la topologie induite par $H$ sur $V$.

En effet, si $\mathbf{u} \in D(A)$, la forme $\mathbf{v} \mapsto a(\mathbf{u}, \mathbf{v})$ se prolonge de façon unique, par densité, en une forme linéaire $L$ continue sur $H$.

Par le théorème de représentation de Riesz, il existe un unique élément de $H$, qu'on note $A \mathbf{u}$, tel que

$$
L(\cdot)=(A \mathbf{u}, \cdot)
$$

c'est à dire

$$
a(\mathbf{u}, \mathbf{v})=(A \mathbf{u}, \mathbf{v}) \quad \forall \mathbf{u} \in D(A), \mathbf{v} \in V .
$$

La correspondance $\mathbf{u} \mapsto A \mathbf{u}$ est linéaire et définit donc un opérateur linéaire $A: D(A) \rightarrow H$ associé au triplet $\{V, H, a\}$. On vérifie aisément que $A$ possède les propriétés suivantes : 
Proposition 1.4. (1) $A \in \mathcal{L}(D(A), H)$ est bijectif.

(2) A est autoadjoint.

On a également le théorème suivant

Théorème 1.5. L'inverse de l'opérateur $A$ est compact dans $H$.

Comme $A$ est autoadjoint, $A^{-1}$ l'est aussi. Comme l'opérateur $A^{-1}$ est de plus compact, il admet une suite de fonctions propres $\mathbf{w}_{j}, j \in \mathbb{N}$, orthogonale et complète dans $H$ et dans $V$,

$$
A^{-1} \mathbf{w}_{j}=\alpha_{j} \mathbf{w}_{j}, \mathbf{w}_{j} \in D(A), \alpha_{j} \in \mathbb{R}^{*}
$$

et donc

$$
A \mathbf{w}_{j}=a_{j} \mathbf{w}_{j} \text { avec } a_{j}=\frac{1}{\alpha_{j}} .
$$

D'autre part, d'après le théorème spectral pour les opérateurs compacts, l'ensemble des valeurs propres de $A^{-1}$ est dénombrable et le seul point d'accumulation possible est zéro, d'où

$$
0<a_{1} \leq a_{2} \leq \ldots a_{j} \underset{j \rightarrow+\infty}{\longrightarrow}+\infty
$$

Ce sont ces fonctions propres que nous allons choisir comme base de Galerkin de $V$ pour démontrer l'existence d'une solution du problème instationnaire.

\section{Le PROBlÈme DE NAVIER-Stokes instationnaire}

On s'intéresse à présent à l'étude du problème de Navier-Stokes instationnaire avec conditions dissipatives naturelles suivant

$$
\left\{\begin{array}{rlrl}
\rho \frac{\partial \mathbf{u}}{\partial t}+\rho(\mathbf{u} \cdot \nabla) \mathbf{u}-\mu \Delta \mathbf{u}+\nabla \mathbf{p} & =0 & & \operatorname{dans}] 0, T[\times \Omega \\
\nabla \cdot \mathbf{u} & =0 & & \text { dans }] 0, T[\times \Omega \\
\mathbf{u} & =0 & & \text { sur }] 0, T\left[\times \Gamma_{l}\right. \\
\mu \nabla \mathbf{u} \cdot \mathbf{n}-\mathbf{p} \mathbf{n} & =-P_{0} \mathbf{n} & & \text { sur }] 0, T\left[\times \Gamma_{0}\right. \\
\mu \nabla \mathbf{u} \cdot \mathbf{n}-\mathbf{p} \mathbf{n} & =-P_{i} \mathbf{n}-R_{i}\left(\int_{\Gamma_{i}} \mathbf{u} \cdot \mathbf{n}\right) \mathbf{n} & & \text { sur }] 0, T\left[\times \Gamma_{i}\right. \\
& & & i=1, \ldots, N \\
\mathbf{u}(x, 0) & =\mathbf{u}_{0}(x) . &
\end{array}\right.
$$

Pour tout $\tilde{\mathbf{u}} \in V$ on a

$$
\rho \int_{\Omega} \frac{\partial \mathbf{u}}{\partial t} \cdot \tilde{\mathbf{u}}+\rho \int_{\Omega}(\mathbf{u} \cdot \nabla \mathbf{u}) \cdot \tilde{\mathbf{u}}+\mu \int_{\Omega} \nabla \mathbf{u}: \nabla \tilde{\mathbf{u}}+\sum_{i=1}^{N} R_{i}\left(\int_{\Gamma_{i}} \mathbf{u} \cdot \mathbf{n}\right)\left(\int_{\Gamma_{i}} \tilde{\mathbf{u}} \cdot \mathbf{n}\right)=-\sum_{i=0}^{N} P_{i}\left(\int_{\Gamma_{i}} \tilde{\mathbf{u}} \cdot \mathbf{n}\right) .
$$

On définit la forme trilinéaire suivante

$$
\begin{gathered}
b: H^{1}(\Omega) \times H^{1}(\Omega) \times H^{1}(\Omega) \rightarrow \mathbb{R} \text { tel que } \\
b(\mathbf{u}, \mathbf{v}, \tilde{\mathbf{u}})=((\mathbf{u} \cdot \nabla) \mathbf{v}, \tilde{\mathbf{u}}) .
\end{gathered}
$$

De plus, on pose

$$
l(\tilde{\mathbf{u}})=-\sum_{i=0}^{N} P_{i} \int_{\Gamma_{i}} \tilde{\mathbf{u}} \cdot \mathbf{n}
$$


pour tout $\tilde{\mathbf{u}} \in H^{1}(\Omega)$.

Le problème que l'on va chercher à résoudre s'écrit : pour

$$
\begin{gathered}
\mathbf{u}_{0} \in V, \\
P_{i} \in L^{\infty}(0, T), i=0, \ldots N, \\
R_{i}>0, i=1, \ldots, N, \text { donnés, }
\end{gathered}
$$

trouver $\mathbf{u} \in L^{\infty}(0, T ; V) \cap L^{2}(0, T ; D(A))$ et $\frac{\partial \mathbf{u}}{\partial t} \in L^{2}(0, T ; H)$ tel que p.p en $t$

$$
\rho\left(\frac{\partial \mathbf{u}}{\partial t}, \tilde{\mathbf{u}}\right)+a(\mathbf{u}, \tilde{\mathbf{u}})+\rho b(\mathbf{u}, \mathbf{u}, \tilde{\mathbf{u}})=l(\tilde{\mathbf{u}}), \quad \forall \tilde{\mathbf{u}} \in V,
$$

avec $\mathbf{u}_{\mid t=0}=\mathbf{u}_{0}$.

Il est à noter que nous nous plaçons ici dans le cadre de solutions fortes. En effet, dans le cas de la dimension 3 , on ne peut pas obtenir d'estimation d'énergie (i. e. de bornes sur la solution dans $L^{2}(0, T ; V) \cap L^{\infty}(0, T ; H)$ qui seraient une première étape afin de montrer l'existence d'une solution faible) car on ne contrôle pas le flux d'énergie cinétique aux entrées et aux sorties du domaine. Ainsi la difficulté pour montrer l'existence d'une solution est d'estimer le terme non linéaire. Pour cela nous allons supposer que

$$
\sup _{\Omega}|\tilde{\mathbf{u}}| \leq c_{1}\|\nabla \tilde{\mathbf{u}}\|_{L^{2}(\Omega)}^{\frac{1}{2}}\|A \tilde{\mathbf{u}}\|_{L^{2}(\Omega)}^{\frac{1}{2}},
$$

pour tout $\tilde{\mathbf{u}} \in D(A)$. L'inégalité (17) est prouvée par exemple dans [6], pages 38-39, pour le cas de conditions de Dirichlet sur toute la frontière du domaine (suffisament régulier), et ceci aussi bien en dimension deux qu'en dimension trois. Elle repose en particulier sur le fait que $D(A)=H^{2}(\Omega) \cap V$. Par contre, dans le cas des conditions aux limites mixtes Dirichlet/Neumann qui nous intéressent, une preuve de (17) est établie dans [3] seulement dans le cas bidimentionnel et pour un domaine $\Omega$ où la paroi latérale et les sections $\Gamma_{i}, i=0, \ldots, N$ sont suffisamment régulières et que les sections $\Gamma_{i}$ et la paroi latérale forment des angles droits. Dans [15] et [20], l'inégalité (17) est admise dans le cas tri-dimensionnel, ce que nous supposerons également. Nous allons également nous appuyer sur

$$
\|\nabla \tilde{\mathbf{u}}\|_{L^{2}(\Omega)} \leq c_{2}\|A \tilde{\mathbf{u}}\|_{L^{2}(\Omega)},
$$

pour tout $\tilde{\mathbf{u}} \in D(A)$, qui elle découle du fait que

$$
\|\nabla \tilde{\mathbf{u}}\|_{L^{2}(\Omega)}^{2} \leq a(\tilde{\mathbf{u}}, \tilde{\mathbf{u}})=(A \tilde{\mathbf{u}}, \tilde{\mathbf{u}}) \leq\|A \tilde{\mathbf{u}}\|_{L^{2}(\Omega)}\|\tilde{\mathbf{u}}\|_{L^{2}(\Omega)} \leq c_{2}\|A \tilde{\mathbf{u}}\|_{L^{2}(\Omega)}\|\nabla \tilde{\mathbf{u}}\|_{L^{2}(\Omega)}
$$

Nous donnons maintenant une estimation valable pour les fonctions de $V$ que nous utiliserons afin d'obtenir des estimations sur la solution.

Proposition 2.1. Si $\mathbf{u} \in V$ alors $\left|\int_{\Gamma_{i}} \mathbf{u} \cdot \mathbf{n}\right| \leq C\|\mathbf{u}\|_{L^{2}(\Omega)}, \forall i=0, \ldots, N$.

Démonstration. Soient $\mathbf{u} \in \mathcal{D}(\bar{\Omega})$ et $v \in \mathcal{D}(\bar{\Omega})$. D'après la formule de Green on a

$$
\int_{\partial \Omega} \mathbf{u} \cdot \mathbf{n} v=\int_{\Omega} \mathbf{u} \nabla v+\int_{\Omega} \nabla \cdot \mathbf{u} v
$$

Par densité de $\mathcal{D}(\bar{\Omega})$ dans $H^{1}(\Omega)$ et l'inégalité de Schwartz, on a $\forall \mathbf{u} \in \mathcal{D}(\bar{\Omega})$ et $\forall v \in H^{1}(\Omega)$

$$
\begin{aligned}
\left|\int_{\partial \Omega} \mathbf{u} \cdot \mathbf{n} v\right| & \leq\left|\int_{\Omega} \mathbf{u} \nabla v\right|+\left|\int_{\Omega} \nabla \cdot \mathbf{u} v\right| \\
& \leq\|\mathbf{u}\|_{L^{2}(\Omega)}\|\nabla v\|_{L^{2}(\Omega)}+\|\nabla \cdot \mathbf{u}\|_{L^{2}(\Omega)}\|v\|_{L^{2}(\Omega)} \\
& \leq\left(\|\mathbf{u}\|_{L^{2}(\Omega)}+\|\nabla \cdot \mathbf{u}\|_{L^{2}(\Omega)}\|v\|_{H^{1}(\Omega)} .\right.
\end{aligned}
$$


Compte tenu de la géométrie du domaine $\Omega$, on peut considérer un élément $w$ de $H^{\frac{1}{2}}(\partial \Omega)$ tel que $w / \Gamma_{i}=1$ et $w_{/ \Gamma_{j}}=0 \forall j \neq i$. Il existe un relèvement $v$ dans $H^{1}(\Omega)$ tel que $v_{/ \partial \Omega}=w$ et $\|v\|_{H^{1}(\Omega)} \leq C\|w\|_{H^{\frac{1}{2}(\partial \Omega)}}$, où $C>0$. Si on considère $\mathbf{u}$ tel que $\mathbf{u} \cdot \mathbf{n}_{/ \Gamma_{l}}=0$, on a

$$
\left|\int_{\Gamma_{i}} \mathbf{u} \cdot \mathbf{n}\right| \leq C_{w}\left(\|\mathbf{u}\|_{L^{2}(\Omega)}+\|\nabla \cdot \mathbf{u}\|_{L^{2}(\Omega)}\right), \quad \forall \mathbf{u} \in \mathcal{D}(\bar{\Omega}), \mathbf{u} \cdot \mathbf{n}_{/ \Gamma_{l}}=0
$$

Par densité, cette inégalité reste vraie pour tout $H_{0, l}^{1}(\Omega)$. En particulier, pour $\mathbf{u}$ tel que $\nabla \cdot \mathbf{u}=0$, on a

$$
\left|\int_{\Gamma_{i}} \mathbf{u} \cdot \mathbf{n}\right| \leq C^{\prime}\|\mathbf{u}\|_{L^{2}(\Omega)}
$$

On établit alors le résultat suivant

Théorème 2.2. Soit $\tilde{P}=\sup _{t} \sum_{i=0}^{N}\left|P_{i}\right|$. Sous les hypothèses (15)-(17), il existe un intervalle de temps sur lequel le problème (16) admet une solution unique. De plus, si on suppose que la condition initiale et $\tilde{P}$ sont suffisamment petites alors la solution du problème existe pour tout $t>0$ et il existe $C>0$ telle que pour tout $t$

$$
\|\nabla \mathbf{u}\|_{L^{2}(\Omega)}^{2}+\sum_{i=1}^{N} R_{i}\left(\int_{\Gamma_{i}} \mathbf{u} \cdot \mathbf{n}\right)^{2} \leq C .
$$

Finalement, la solution est unique et dépend continuement des données.

Plus précisément, soient $\left(\mathbf{u}_{1}, \mathbf{u}_{2}\right)$ deux solutions associées aux données $\left(\mathbf{u}_{0}^{1}, P_{i}^{1}\right), i=1 \ldots N$ et $\left(\mathbf{u}_{0}^{2}, P_{i}^{2}\right), i=$ $1 \ldots N$, on $a$

$$
\left\|\mathbf{u}^{1}-\mathbf{u}^{2}\right\|_{L^{2}(\Omega)}^{2} \leq\left\|\mathbf{u}_{0}^{1}-\mathbf{u}_{0}^{2}\right\|_{L^{2}(\Omega)}^{2}+C \max _{1 \leq i \leq N}\left|P_{i}^{1}-P_{i}^{2}\right| \int_{0}^{t} \exp \left(C \int_{u}^{t}\left(\left(\sup _{\Omega}\left|\mathbf{u}_{2}\right|\right)^{2}+\left\|\nabla \mathbf{u}_{1}\right\|_{L^{2}(\Omega)}^{4}\right)\right) d u .
$$

Démonstration. Le schéma de la preuve du théorème 2.2 est le suivant : on obtient tout d'abord des estimations a priori vérifiées par l'approximation de Galerkin $\mathbf{u}_{m}$ de la solution de (16) qui nous permettront de passer à la limite lorsque $m$ tend vers $+\infty$, prouvant ainsi l'existence d'une solution locale en temps. Ensuite, si les données initiales sont suffisamment petites, on prouve de nouvelles estimations a priori assurant l'existence d'une solution pour tout $t>0$. Finalement, on prouve que la solution limite est unique et dépend continuement des données.

Pour tout $m \in \mathbb{N}$, on définit l'approximation de Galerkin $\mathbf{u}_{m}(t)$ comme solution dans $\operatorname{Vect}\left\{\mathbf{w}_{1}, \ldots, \mathbf{w}_{m}\right\}$ du système non linéaire suivant

$$
\left\{\begin{array}{l}
\rho\left(\frac{\partial \mathbf{u}_{m}}{\partial t}, \tilde{\mathbf{u}}\right)+a\left(\mathbf{u}_{m}, \tilde{\mathbf{u}}\right)+\rho b\left(\mathbf{u}_{m}, \mathbf{u}_{m}, \tilde{\mathbf{u}}\right)=l(\tilde{\mathbf{u}}), \quad \forall \tilde{\mathbf{u}} \in \operatorname{Vect}\left\{\mathbf{w}_{1}, \ldots, \mathbf{w}_{m}\right\} \\
\int_{\Omega}\left(\mathbf{u}_{m}(0)-\mathbf{u}_{0}\right) \tilde{\mathbf{u}}=0, \quad \forall \tilde{\mathbf{u}} \in \operatorname{Vect}\left\{\mathbf{w}_{1}, \ldots, \mathbf{w}_{m}\right\}
\end{array}\right.
$$

Ce système différentiel de $m$ équations à $m$ inconnues admet une solution unique sur un intervalle $\left[0, t_{m}\right]$.

Estimations a priori et existence locale

On choisit comme fonction test dans la formulation variationnelle $(20), \tilde{\mathbf{u}}=A \mathbf{u}_{m}$, ce qui est possible car $A \mathbf{u}_{m} \in \operatorname{Vect}\left\{\mathbf{w}_{1}, \ldots, \mathbf{w}_{m}\right\}$. On a alors

$$
\rho\left(\frac{\partial \mathbf{u}_{m}}{\partial t}, A \mathbf{u}_{m}\right)+a\left(\mathbf{u}_{m}, A \mathbf{u}_{m}\right)+\rho b\left(\mathbf{u}_{m}, \mathbf{u}_{m}, A \mathbf{u}_{m}\right)=c\left(t, A \mathbf{u}_{m}\right)
$$


Or on a

$$
\rho\left(\frac{\partial \mathbf{u}_{m}}{\partial t}, A \mathbf{u}_{m}\right)=\rho \mu \frac{1}{2} \frac{d}{d t}\left\|\nabla \mathbf{u}_{m}\right\|_{L^{2}(\Omega)}^{2}+\rho \frac{1}{2} \sum_{i=1}^{N} R_{i} \frac{d}{d t}\left(\int_{\Gamma_{i}} \mathbf{u}_{m} \cdot \mathbf{n}\right)^{2}
$$

et

$$
a\left(\mathbf{u}_{m}, A \mathbf{u}_{m}\right)=\left(A \mathbf{u}_{m}, A \mathbf{u}_{m}\right)=\left\|A \mathbf{u}_{m}\right\|_{L^{2}(\Omega)}^{2}
$$

il s'ensuit

$$
\frac{1}{2} \rho \mu \frac{d}{d t}\left\|\nabla \mathbf{u}_{m}\right\|_{L^{2}(\Omega)}^{2}+\frac{1}{2} \rho \sum_{i=1}^{N} R_{i} \frac{d}{d t}\left(\int_{\Gamma_{i}} \mathbf{u}_{m} \cdot \mathbf{n}\right)^{2}+\left\|A \mathbf{u}_{m}\right\|_{L^{2}(\Omega)}^{2}=-\rho b\left(\mathbf{u}_{m}, \mathbf{u}_{m}, A \mathbf{u}_{m}\right)-\sum_{i=0}^{N} P_{i} \int_{\Gamma_{i}} A \mathbf{u}_{m} \cdot \mathbf{n} .
$$

A ce niveau de la preuve, on fait appel à l'inégalité d'interpolation (17) pour tout $\tilde{\mathbf{u}} \in D(A)$ et à l'inégalité de trace suivante (voir proposition 2.1), pour tout $\tilde{\mathbf{u}} \in \operatorname{Vect}\left\{\mathbf{w}_{1}, \ldots, \mathbf{w}_{m}\right\}$ puisque $A \tilde{\mathbf{u}} \in V$,

$$
\left|\sum_{i=1}^{n} P_{i} \int_{\Gamma_{i}} A \tilde{\mathbf{u}} \cdot \mathbf{n}\right| \leq c_{3} \tilde{P}\|A \tilde{\mathbf{u}}\|_{L^{2}(\Omega)}
$$

où $\tilde{P}=\sup _{t \geq 0} \sum_{i=1}^{n}\left|P_{i}\right|$.

Grâce à ces deux inégalités, on a

$$
\begin{gathered}
\frac{1}{2} \rho \mu \frac{d}{d t}\left\|\nabla \mathbf{u}_{m}\right\|_{L^{2}(\Omega)}^{2}+\frac{1}{2} \rho \sum_{i=1}^{N} R_{i} \frac{d}{d t}\left(\int_{\Gamma_{i}} \mathbf{u}_{m} \cdot \mathbf{n}\right)^{2}+\left\|A \mathbf{u}_{m}\right\|_{L^{2}(\Omega)}^{2} \leq \rho\left|b\left(\mathbf{u}_{m}, \mathbf{u}_{m}, A \mathbf{u}_{m}\right)\right|+c_{3} \tilde{P}\left\|A \mathbf{u}_{m}\right\|_{L^{2}(\Omega)} \\
\leq \rho \sup _{\Omega}\left|\mathbf{u}_{m}\right|\left\|\nabla \mathbf{u}_{m}\right\|_{L^{2}(\Omega)}\left\|A \mathbf{u}_{m}\right\|_{L^{2}(\Omega)}+c_{3} \tilde{P}\left\|A \mathbf{u}_{m}\right\|_{L^{2}(\Omega)} \\
\leq \rho c_{1}\left\|\nabla \mathbf{u}_{m}\right\|_{L^{2}(\Omega)}^{\frac{3}{2}}\left\|A \mathbf{u}_{m}\right\|_{L^{2}(\Omega)}^{\frac{3}{2}}+c_{3} \tilde{P}\left\|A \mathbf{u}_{m}\right\|_{L^{2}(\Omega)}
\end{gathered}
$$

En appliquant l'inégalité de Young

$$
a b \leq \frac{a^{p}}{p}+\frac{b^{q}}{q} \text { avec } \frac{1}{p}+\frac{1}{q}=1
$$

aux deux termes du membre de droite, respectivement avec $p=4$ et $q=\frac{4}{3}$ puis $p=2$ et $q=2$, on a alors

$$
\frac{d}{d t}\left\|\nabla \mathbf{u}_{m}\right\|_{L^{2}(\Omega)}^{2}+\frac{1}{\mu} \sum_{i=1}^{N} R_{i} \frac{d}{d t}\left(\int_{\Gamma_{i}} \mathbf{u}_{m} \cdot \mathbf{n}\right)^{2}+\frac{1}{\rho \mu}\left\|A \mathbf{u}_{m}\right\|_{L^{2}(\Omega)}^{2} \leq \frac{4 \rho^{3} c_{1}^{4}}{\mu}\left\|\nabla \mathbf{u}_{m}\right\|_{L^{2}(\Omega)}^{6}+\frac{4 c_{3}^{2}}{\rho \mu} \tilde{P}^{2} .
$$

A ce niveau de la preuve, on considère

$$
\begin{gathered}
\phi(t)=\left\|\nabla \mathbf{u}_{m}\right\|_{L^{2}(\Omega)}^{2}+\frac{1}{\mu} \sum_{i=1}^{N} R_{i}\left(\int_{\Gamma_{i}} \mathbf{u}_{m} \cdot \mathbf{n}\right)^{2}, \\
\psi(t)=\frac{1}{\rho \mu} \int_{0}^{t}\left\|A \mathbf{u}_{m}\right\|_{L^{2}(\Omega)}^{2}
\end{gathered}
$$

et

$$
f(t)=\frac{4 c_{3}^{2} \tilde{P}^{2}}{\rho \mu}
$$


L'inégalité ci-dessus s'écrit alors

$$
\frac{d}{d t} \phi(t)+\psi(t) \leq \frac{4 c_{1}^{4} \rho^{3}}{\mu} \phi^{3}(t)+f(t)
$$

Par ailleurs, comme on a

$$
\int_{\Omega}\left(\mathbf{u}_{m}(0)-\mathbf{u}_{0}\right) \tilde{\mathbf{u}}=0, \quad \forall \tilde{\mathbf{u}} \in \operatorname{Vect}\left\{\mathbf{w}_{1}, \ldots, \mathbf{w}_{m}\right\}
$$

et en choisissant $\tilde{\mathbf{u}}=A \mathbf{u}_{m}(0)$ on obtient

$$
\left(\mathbf{u}_{m}(0), A \mathbf{u}_{m}(0)\right)=\left(\mathbf{u}_{0}, A \mathbf{u}_{m}(0)\right)
$$

c'est à dire

$$
a\left(\mathbf{u}_{m}(0), \mathbf{u}_{m}(0)\right)=a\left(\mathbf{u}_{m}(0), \mathbf{u}_{0}\right)
$$

or

Ainsi

$$
\mu\left\|\nabla \mathbf{u}_{m}(0)\right\|_{L^{2}(\Omega)}^{2} \leq a\left(\mathbf{u}_{m}(0), \mathbf{u}_{m}(0)\right)=a\left(\mathbf{u}_{m}(0), \mathbf{u}_{0}\right) \leq C\left\|\nabla \mathbf{u}_{m}(0)\right\|_{L^{2}(\Omega)}\left\|\nabla \mathbf{u}_{0}\right\|_{L^{2}(\Omega)}
$$

et donc

$$
\mu\left\|\nabla \mathbf{u}_{m}(0)\right\|_{L^{2}(\Omega)} \leq C\left\|\nabla \mathbf{u}_{0}\right\|_{L^{2}(\Omega)}
$$

$$
\phi(0)=\left\|\nabla \mathbf{u}_{m}(0)\right\|_{L^{2}(\Omega)}^{2}+\frac{1}{\mu} \sum_{i=1}^{N} R_{i}\left(\int_{\Gamma_{i}} \mathbf{u}_{m}(0) \cdot \mathbf{n}\right)^{2} \leq \frac{C}{\mu}\left\|\nabla \mathbf{u}_{0}\right\|_{L^{2}(\Omega)}^{2} .
$$

Ainsi, par le lemme de comparaison pour les inéquations différentielles [14], il existe $t^{*} \leq t_{m}$ ne dépendant que de $\mathbf{u}_{0}$ et une fonction $F\left(t,\left\|\nabla \mathbf{u}_{0}\right\|_{L^{2}(\Omega)}\right)$ solution de l'équation différentielle $F^{\prime}=\frac{4 c_{1}^{4} \rho^{3}}{\mu} F^{3}+f, F(0)=$ $\frac{C}{\mu}\left\|\nabla \mathbf{u}_{0}\right\|_{L^{2}(\Omega)}^{2}$ sur $\left[0, t^{*}\left[\right.\right.$, ainsi que $\tilde{F}\left(t,\left\|\nabla \mathbf{u}_{0}\right\|_{L^{2}(\Omega)}\right)$ tel que, pour tout $t \in\left[0, t^{*}[\right.$ on a

$$
\begin{gathered}
\phi(t) \leq F(t), \\
\text { et } \int_{0}^{t} \psi(\tau) d \tau \leq \tilde{F}(t),
\end{gathered}
$$

c'est à dire

$$
\left\|\nabla \mathbf{u}_{m}\right\|_{L^{2}(\Omega)}^{2}+\frac{1}{\mu} \sum_{i=1}^{N} R_{i}\left(\int_{\Gamma_{i}} \mathbf{u}_{m} \cdot \mathbf{n}\right)^{2} \leq F(t)
$$

et

$$
\frac{1}{\rho \mu} \int_{0}^{t}\left\|A \mathbf{u}_{m}\right\|_{L^{2}(\Omega)}^{2} \leq \tilde{F}(t) .
$$

Grâce à ces estimations, on peut également déduire une estimation pour $\int_{0}^{t}\left\|\frac{\partial \mathbf{u}_{m}}{\partial \tau}\right\|_{L^{2}(\Omega)}^{2} d \tau$. En effet, en choisissant $\tilde{\mathbf{u}}=\frac{\partial \mathbf{u}_{m}}{\partial t}$ dans $(20)$ on a

$$
\begin{aligned}
\rho\left\|\frac{\partial \mathbf{u}_{m}}{\partial t}\right\|_{L^{2}(\Omega)}^{2} & =-\rho \int_{\Omega}\left(\mathbf{u}_{m} \cdot \nabla \mathbf{u}_{m}\right) \frac{\partial \mathbf{u}_{m}}{\partial t}-\left(A \mathbf{u}_{m}, \frac{\partial \mathbf{u}_{m}}{\partial t}\right)-\sum_{i=0}^{N} P_{i}\left(\int_{\Gamma_{i}} \frac{\partial \mathbf{u}_{m}}{\partial t} \cdot \mathbf{n}\right) \\
& \leq\left(\rho\left\|\mathbf{u}_{m}\right\|_{L^{\infty}(\Omega)}\left\|\nabla \mathbf{u}_{m}\right\|_{L^{2}(\Omega)}+\left\|A \mathbf{u}_{m}\right\|_{L^{2}(\Omega)}+C \tilde{P}\right)\left\|\frac{\partial \mathbf{u}_{m}}{\partial t}\right\|_{L^{2}(\Omega)} \\
& \leq\left(\rho c_{1}\left\|\nabla \mathbf{u}_{m}\right\|_{L^{2}(\Omega)}^{\frac{3}{2}}\left\|A \mathbf{u}_{m}\right\|_{L^{2}(\Omega)}^{\frac{1}{2}}+\left\|A \mathbf{u}_{m}\right\|_{L^{2}(\Omega)}+c \tilde{P}\right)\left\|\frac{\partial \mathbf{u}_{m}}{\partial t}\right\|_{L^{2}(\Omega)},
\end{aligned}
$$


d'où

$$
\left\|\frac{\partial \mathbf{u}_{m}}{\partial t}\right\|_{L^{2}(\Omega)} \leq \frac{1}{\rho}\left(\rho c_{1}\left\|\nabla \mathbf{u}_{m}\right\|_{L^{2}(\Omega)}^{\frac{3}{2}}\left\|A \mathbf{u}_{m}\right\|_{L^{2}(\Omega)}^{\frac{1}{2}}+\left\|A \mathbf{u}_{m}\right\|_{L^{2}(\Omega)}+c \tilde{P}\right),
$$

et donc, grâce aux estimations (23)-(24), il existe une fonction $G$ dans $L^{1}\left(0, t^{*}\right)$ ne dépendant que des données telle que

$$
\int_{0}^{t}\left\|\frac{\partial \mathbf{u}_{m}}{\partial \tau}\right\|_{L^{2}(\Omega)}^{2} d \tau \leq G(t)
$$

\section{Passage à la limite}

Pour tout $t \leq t^{*}$, on a les estimations suivantes :

$$
\begin{array}{r}
\left\|\nabla \mathbf{u}_{m}\right\|_{L^{2}(\Omega)}^{2} \leq C F\left(t,\left\|\nabla \mathbf{u}_{0}\right\|_{L^{2}(\Omega)}\right), \\
\int_{0}^{t}\left\|A \mathbf{u}_{m}\right\|_{L^{2}(\Omega)}^{2} \leq C \tilde{F}\left(t,\left\|\nabla \mathbf{u}_{0}\right\|_{L^{2}(\Omega)}\right), \\
\int_{0}^{t}\left\|\frac{\partial \mathbf{u}_{m}}{\partial \tau}\right\|_{L^{2}(\Omega)}^{2} \leq G\left(t,\left\|\nabla \mathbf{u}_{0}\right\|_{L^{2}(\Omega)}\right) .
\end{array}
$$

Ce qui permet de déduire que $\mathbf{u}_{m}$ est bornée dans $L^{\infty}\left(0, t^{*} ; V\right) \cap L^{2}\left(0, t^{*} ; D(A)\right)$ et que $\frac{\partial \mathbf{u}_{m}}{\partial t}$ est bornée dans $L^{2}\left(0, t^{*} ; H\right)$.

Grâce aux estimations (25), on peut extraire une sous suite qu'on notera également $\mathbf{u}_{m}$ telle que

$$
\left\{\begin{array}{l}
\mathbf{u}_{m} \rightarrow \mathbf{u} \text { faible }{ }^{*} \text { dans } L^{\infty}\left(0, t^{*} ; V\right) \\
\text { et } \\
\frac{\partial \mathbf{u}_{m}}{\partial t} \rightarrow \frac{\partial \mathbf{u}}{\partial t} \text { faiblement dans } L^{2}\left(0, t^{*} ; H\right)
\end{array}\right.
$$

Par ailleurs, grâce au lemme de compacité énoncé dans [16], page 57, on peut extraire de $\mathbf{u}_{m}$ une sous suite qu'on note également $\mathbf{u}_{m}$ telle que

$$
\mathbf{u}_{m} \underset{m \rightarrow+\infty}{\longrightarrow} \mathbf{u} \text { dans } L^{2}\left(0, t^{*} ; L^{2}(\Omega)\right) .
$$

On peut alors passer à la limite dans les termes de la formulation pour montrer que u vérifie (16) localement en temps.

\section{Existence globale}

On a déjà établit que

$$
\begin{aligned}
& \frac{\mu \rho}{2} \frac{d}{d t}\left\|\nabla \mathbf{u}_{m}\right\|_{L^{2}(\Omega)}^{2}+\frac{\rho}{2} \sum_{i=1}^{N} R_{i} \frac{d}{d t}\left(\int_{\Gamma_{i}} \mathbf{u}_{m} \cdot \mathbf{n}\right)^{2}+\left\|A \mathbf{u}_{m}\right\|_{L^{2}(\Omega)}^{2} \leq \\
& \rho \sup _{\Omega}\left|\mathbf{u}_{m}\right|\left\|\nabla \mathbf{u}_{m}\right\|_{L^{2}(\Omega)}\left\|A \mathbf{u}_{m}\right\|_{L^{2}(\Omega)}+c_{3} \tilde{P}\left\|A \mathbf{u}_{m}\right\|_{L^{2}(\Omega)} .
\end{aligned}
$$

La combinaison des inégalités (17) et (18) donne

$$
\sup _{\Omega}|\mathbf{u}| \leq c_{1} c_{2}^{\frac{1}{2}}\|A \mathbf{u}\|_{L^{2}(\Omega)}
$$


Il en découle que

$$
\begin{aligned}
& \frac{\mu \rho}{2} \frac{d}{d t}\left\|\nabla \mathbf{u}_{m}\right\|_{L^{2}(\Omega)}^{2}+\frac{\rho}{2} \sum_{i=1}^{N} R_{i} \frac{d}{d t}\left(\int_{\Gamma_{i}} \mathbf{u}_{m} \cdot \mathbf{n}\right)^{2}+\left\|A \mathbf{u}_{m}\right\|_{L^{2}(\Omega)}^{2} \\
& \leq \rho c_{1} c_{2}^{\frac{1}{2}}\left\|A \mathbf{u}_{m}\right\|_{L^{2}(\Omega)}^{2}\left\|\nabla \mathbf{u}_{m}\right\|_{L^{2}(\Omega)}+c_{3} \tilde{P}\left\|A \mathbf{u}_{m}\right\|_{L^{2}(\Omega)} \\
& \leq \rho c_{1} c_{2}^{\frac{1}{2}}\left\|A \mathbf{u}_{m}\right\|_{L^{2}(\Omega)}^{2}\left\|\nabla \mathbf{u}_{m}\right\|_{L^{2}(\Omega)}+\frac{c_{3}^{2} \tilde{P}^{2}}{2}+\frac{1}{2}\left\|A \mathbf{u}_{m}\right\|_{L^{2}(\Omega)}^{2},
\end{aligned}
$$

d'où

$$
\frac{d}{d t}\left\|\nabla \mathbf{u}_{m}\right\|_{L^{2}(\Omega)}^{2}+\frac{1}{\mu} \sum_{i=1}^{N} R_{i} \frac{d}{d t}\left(\int_{\Gamma_{i}} \mathbf{u}_{m} \cdot \mathbf{n}\right)^{2}+\left(\frac{1-2 \rho c_{1} c_{2}^{\frac{1}{2}}\left\|\nabla \mathbf{u}_{m}\right\|_{L^{2}(\Omega)}}{\rho \mu}\right)\left\|A \mathbf{u}_{m}\right\|_{L^{2}(\Omega)}^{2} \leq \frac{c_{3}^{2} \tilde{P}^{2}}{4 \mu \rho} .
$$

Ainsi, en utilisant l'inégalité (18) et le fait que $\left|\int_{\Gamma_{i}} \mathbf{v} \cdot \mathbf{n}\right| \leq C\|\nabla \mathbf{v}\|_{L^{2}(\Omega)}$ pour $\mathbf{v} \in H^{1}(\Omega)$, on obtient une inégalité différentielle du type

$$
\frac{d}{d t} \phi+A(1-B \sqrt{\phi}) \phi \leq \frac{c_{3}^{2} \tilde{P}^{2}}{4 \mu \rho}
$$

valable si $\sqrt{\phi} \leq 1 / B$ avec $\phi=\left\|\nabla \mathbf{u}_{m}\right\|_{L^{2}(\Omega)}^{2}+\sum_{i=1}^{N} \frac{R_{i}}{\mu}\left(\int_{\Gamma_{i}} \mathbf{u}_{m} \cdot \mathbf{n}\right)^{2}$. Par conséquent, en supposant que $\sqrt{\phi(0)} \leq$ $\frac{1}{2 B}$ et que $\frac{c_{3}^{2} \tilde{P}^{2}}{4 \mu \rho} \leq \frac{A \mu^{2}}{4 B^{2}}$, il est facile de vérifier que $\sqrt{\phi(t)} \leq \frac{1}{2 B}$ pour tout $t$. Ceci clôt la preuve d'existence globale pour des données petites.

\section{Unicité et dépendance continue par rapport aux données}

On considère maintenant la question de l'unicité et de la dépendance continue par rapport aux données. On prouve tout d'abord qu'il existe un intervalle de temps où la solution dépend continuement des données. On prouvera ensuite l'unicité à l'aide des mêmes estimations. On note $\mathbf{u}_{1}$ une solution associée aux données $\left(\mathbf{u}_{0}^{1}, P_{i}^{1}\right) i=0, \ldots, N$ et de la même manière $\mathbf{u}_{2}$ une solution associée aux données $\left(\mathbf{u}_{0}^{2}, P_{i}^{2}\right) i=0, \ldots, N$. Soient

$$
\mathbf{w}=\mathbf{u}_{1}-\mathbf{u}_{2} \quad \text { et } \quad \delta \tilde{P}=\max _{i}\left|P_{i}^{1}-P_{i}^{2}\right| .
$$

On a

et

$$
\rho\left(\frac{\partial \mathbf{u}_{1}}{\partial t}, \tilde{\mathbf{u}}\right)+a\left(\mathbf{u}_{1}, \tilde{\mathbf{u}}\right)+\rho b\left(\mathbf{u}_{1}, \mathbf{u}_{1}, \tilde{\mathbf{u}}\right)=l(\tilde{\mathbf{u}})
$$

$$
\rho\left(\frac{\partial \mathbf{u}_{2}}{\partial t}, \tilde{\mathbf{u}}\right)+a\left(\mathbf{u}_{2}, \tilde{\mathbf{u}}\right)+\rho b\left(\mathbf{u}_{2}, \mathbf{u}_{2}, \tilde{\mathbf{u}}\right)=l(\tilde{\mathbf{u}}) .
$$

On soustrait les deux équations, et on choisit $\tilde{\mathbf{u}}=\mathbf{w}$, on a

$$
\begin{aligned}
\rho \frac{1}{2} \frac{d}{d t}\|\mathbf{w}\|_{L^{2}(\Omega)}^{2}+\mu\|\nabla \mathbf{w}\|_{L^{2}(\Omega)}^{2}+\sum_{i=1}^{N} R_{i}\left(\int_{\Gamma_{i}} \mathbf{w} \cdot \mathbf{n}\right)^{2} & =-\rho\left(b\left(\mathbf{u}_{1}, \mathbf{u}_{1}, \mathbf{w}\right)-b\left(\mathbf{u}_{2}, \mathbf{u}_{2}, \mathbf{w}\right)\right) \\
& +\sum_{i=0}^{N}\left(P_{i}^{2}-P_{i}^{1}\right) \int_{\Gamma_{i}} \mathbf{w} \cdot \mathbf{n}
\end{aligned}
$$

Or

$$
b\left(\mathbf{u}_{1}, \mathbf{u}_{1}, \mathbf{w}\right)-b\left(\mathbf{u}_{2}, \mathbf{u}_{2}, \mathbf{w}\right)=b\left(\mathbf{u}_{2}, \mathbf{w}, \mathbf{w}\right)+b\left(\mathbf{w}, \mathbf{u}_{1}, \mathbf{w}\right)
$$


et

$$
\left|b\left(\mathbf{u}_{2}, \mathbf{w}, \mathbf{w}\right)\right| \leq \sup _{\Omega}\left|\mathbf{u}_{2}\right|\|\nabla \mathbf{w}\|_{L^{2}(\Omega)}\|\mathbf{w}\|_{L^{2}(\Omega)}
$$

et par l'inégalité de Hölder on a

$$
\left|b\left(\mathbf{w}, \mathbf{u}_{1}, \mathbf{w}\right)\right| \leq\|\mathbf{w}\|_{L^{6}(\Omega)}\left\|\nabla \mathbf{u}_{1}\right\|_{L^{2}(\Omega)}\|\mathbf{w}\|_{L^{3}(\Omega)} .
$$

Rappelons que, grâce aux injections de Sobolev, on a les inégalités suivantes pour $d=2,3$

$$
\|\mathbf{v}\|_{L^{6}(\Omega)} \leq C\|\nabla \mathbf{v}\|_{L^{2}(\Omega)}
$$

et

$$
\|\mathbf{v}\|_{L^{3}(\Omega)} \leq C\|\mathbf{v}\|_{L^{2}(\Omega)}^{\frac{1}{2}}\|\nabla \mathbf{v}\|_{L^{2}(\Omega)}^{\frac{1}{2}} .
$$

Ces estimations, les inégalités de Young et l'inégalité de Poincaré, impliquent

$$
\begin{aligned}
\rho \frac{d}{d t}\|\mathbf{w}\|_{L^{2}(\Omega)}^{2}+2 \mu\|\nabla \mathbf{w}\|_{L^{2}(\Omega)}^{2} & \leq C\left(\sup _{\Omega}\left|\mathbf{u}_{2}\right|\|\mathbf{w}\|_{L^{2}(\Omega)}\right)^{2}+\frac{\mu}{3}\|\nabla \mathbf{w}\|_{L^{2}(\Omega)}^{2} \\
& +C\left\|\nabla \mathbf{u}_{1}\right\|_{L^{2}(\Omega)}^{4}\|\mathbf{w}\|_{L^{2}(\Omega)}^{2}+\frac{\mu}{3}\|\nabla \mathbf{w}\|_{L^{2}(\Omega)}^{2} \\
& +C \delta \tilde{P}^{2}+\frac{\mu}{3}\|\nabla \mathbf{w}\|_{L^{2}(\Omega)}^{2} .
\end{aligned}
$$

Ainsi, on a finalement

$$
\rho \frac{d}{d t}\|\mathbf{w}\|_{L^{2}(\Omega)}^{2}+\mu\|\nabla \mathbf{w}\|_{L^{2}(\Omega)}^{2} \leq C\left(\left(\sup _{\Omega}\left|\mathbf{u}_{2}\right|\right)^{2}+\left\|\nabla \mathbf{u}_{1}\right\|_{L^{2}(\Omega)}^{4}\right)\|\mathbf{w}\|_{L^{2}(\Omega)}^{2}+C \delta \tilde{P}^{2} .
$$

Or $\left(\sup _{\Omega}\left|\mathbf{u}_{2}\right|\right)^{2}+\left\|\nabla \mathbf{u}_{1}\right\|_{L^{2}(\Omega)}^{4}$ est intégrable en temps car $\left.\mathbf{u}_{i} \in L^{2}\left(0, t^{*} ; D(A)\right) \cap L^{\infty}\left(0, t^{*} ; V\right)\right)$ et $D(A) \subset L^{\infty}(\Omega)$ d'après les hypothèses faites sur l'opérateur $A$. Par conséquent, appliquant le lemme de Gronwall, cela entraine (19). En particulier, dès que $\left.\mathbf{u}_{1}\right|_{t=0}=\left.\mathbf{u}_{2}\right|_{t=0}$

$$
\|\mathbf{w}\|_{L^{2}(\Omega)}^{2} \leq C_{T} \delta \tilde{P}^{2} .
$$

Ainsi si $\delta \tilde{P}=0$ alors $\mathbf{w}=0$, ce qui entraine que la solution est unique.

Remarque 2.3. Comme $\mathbf{w}$ est à divergence nulle, on a pour $i=0, \ldots, N$,

$$
\left|\int_{\Gamma_{i}} \mathbf{w} \cdot \mathbf{n}\right| \leq C\|\mathbf{w}\|_{L^{2}(\Omega)} .
$$

Cette inégalité implique en particulier que le débit sur les sections $\Gamma_{i}$ est une fonction continue des données.

\section{Conclusion}

Nous avons donc montré l'existence et l'unicité, locale en temps, de solutions ainsi que l'existence globale pour des données petites pour le système de Navier-Stokes avec des conditions aux limites, qu'on peut qualifier de naturelles dissipatives. Si l'on n'avait pas pris en compte le terme dissipatif $\sum_{i=1}^{N} R_{i}\left(\int_{\Gamma_{i}} \mathbf{u} \cdot \mathbf{n}\right)\left(\int_{\Gamma_{i}} \tilde{\mathbf{u}} \cdot \mathbf{n}\right)$ lors de la définition de l'opérateur $A$, et qu'on l'avait juste défini en l'associant à la forme bilinéaire sur $V$

$$
\int_{\Omega} \nabla \mathbf{u}: \nabla \tilde{\mathbf{u}}
$$


alors, d'une manière analogue à [20], nous aurions obtenu un résultat d'existence locale en temps mais sous une condition supplémentaire du type $\mu-C \max _{1<i<N} R_{i} \geq 0$, ainsi qu'un résultat d'existence globale pour des données petites sous la même condition. Rappelons que les résistances $R_{i}$ correspondent aux résistances globales des sous arbres $\tilde{\Omega}_{i}$ dans lesquels on suppose que la loi de Poiseuille est satisfaite. La condition supplémentaire $\mu-C \max _{1 \leq i \leq N} R_{i} \geq 0$, dont nous nous sommes affranchie ici, est délicate à interpréter suivant la nature du domaine $\Omega$. En effet, dans le cas de l'arbre bronchique humain et en se basant sur les données du modèle de Weibel [23], plus on recule la zone où on décide de tronquer, c'est à dire, plus on réduit le nombre de générations de bronchioles dans la partie distale du modèle et plus les résistances $R_{i}$ qui caractérisent les sous-arbres sont grandes (i. e. plus le domaine de calcul $\Omega$ est grand, plus les $R_{i}$ sont grandes). Au contraire, si le domaine $\Omega$ est un simple tube, on observe que la résistance $R$ de la partie condensée diminue avec la longueur de celle-ci (i. e. plus le domaine de calcul $\Omega$ est grand, plus $R$ est petite). Néanmoins, la condition sous laquelle nous obtenons l'existence globale en temps fait également intervenir les résistances $R_{i}$. En effet on a que $R_{i}\left(\int_{\Gamma_{i}} \mathbf{u}_{0} \cdot \mathbf{n}\right)^{2}$ doit être suffisamment petit. Cependant cette condition fait également intervenir le flux de la condition initiale sur les sorties de l'arbre et donc lorque $R_{i}$ est grande nous obtenons malgré tout l'existence globale, à condition (entre autre) que les flux de la vitesse initiale à travers $\Gamma_{i}$ soient suffisamment petits.

\section{REFERENCES}

[1] I. Babuška, The finite element method with Lagrangian multipliers, Numer. Math., 20, 179-192, 1973.

[2] L. Baffico, C. Grandmont, B. Maury, A. Soualah, Papier en cours de préparation.

[3] H. Blum, R. Rannacher, On the boundary value problem of the biharmonic operator on domains with angular corners, Math. Meth. in the Appl. Sci., 2, 556-581, 1980.

[4] J. M. Boland, R. A. Nicolaides, Stability of finite element under divergence constraints, SIAM J. Number. Anal., Vol. 20, No. 4, 722-731, 1983.

[5] F. Brezzi, On the existence, uniquness and approximation of saddle-point problems arising from lagrange multipliers, RAIRO, Anal. Num. R2, 129-151, 1974.

[6] P. Constantin, C. Foias Navier-Stokes equations, Chicago Lectures in Mathematics, University of Chicago Press, Chicago, 1988.

[7] C. Croce, R. Fodil, M. Durand, G. Sbirlea-Apiou, G. Caillibotte, J.-F. Papon, J.-R. Blondeau, A. Coste, D. Isabey, B. Louis, In vitro experiments and numerical simulations of airflow in realistic nasal airway geometry Annals of biomedical engineering, (2006) vol. 34, no6, pp. 997-1007.

[8] J.W. De Backer, W.G. Vos, A. Devolder, S.L. Verhulst, P. Germonpre, F.L. Wuyts, P.M. Parizel, W. De Backer Computational fluid dynamics can detect changes in airway resistance in asthmatics after acute bronchodilation, Journal of Biomechanics 41 (2008) 106-113.

[9] C. Fetita, S. Mancini, D. Perchet, F. Prêteux, M. Thiriet, and L. Vial, An image based computational model of oscillatory flow in the proximal part of tracheobronchial trees, Comput. Meth. Biomech. Biomed. Eng., 8 (2005), pp. $27-293$.

[10] V. Girault, H. Lopez, B. Maury, One Time-step Finite Element discretization of the Equation of Motion of Two-fluid Flows, Numerical Methods for Differential Equations, Vol. 22. 3, 680-707, 2006.

[11] V. Girault, P.-A. Raviart, Finite Element Approximation of the Navier-Stokes Equations, Lecture Notes in Mathematics, Vol 749 Springer Verlag, Berlin, 1979.

[12] C. Grandmont, B. Maury, N. Meunier, A viscoelastic model with non-local damping. Application to the human lungs, ESAIM : M2AN, Vol. 40, No. 1, 201-224, 2006.

[13] C. Grandmont, B. Maury, A. Soualah, Multiscale modelling of the respiratory track : a theoretical framework, à paraitre dans ESAIM : Proceedings, 2007.

[14] J. G. Heywood, The Navier-Stokes Equations : On the Existence, Regularity and Decay of Solutions, Indiana Univ. Math. J., 29, 636-681, 1980 .

[15] J. G. Heywood, R. Rannacher, S. Turek, Artificial boundaries and flux and pressure conditions for the incompressible NavierStokes equations, Int. J. Numer. Math. Fluids, 22, 325-352, 1996.

[16] J. L. Lions, Quelques méthodes de résolution des problèmes aux limites non linéaires, Dunod, 2002.

[17] B. Maury, N. Meunier, A. Soualah, L. Vial, Outlet dissipative conditions for air flow in the bronchial tree, ESAIM : Proceedings, Vol. 14, 201-212, 2005 .

[18] M. S. Olufsen, Structured tree outflow condition for blood flow in larger systemic arteries, Am. J. Physiol. Heart Circ. Physiol., 276, 257-268, 1999.

[19] G. Prodi, Theoremi di tipo locale per il sistema di Navier-Stokes e stabilita della soluzioni stazionarie, Rend. Sem. Mat. Univ. Padova, 32, 374-397, 1962. 
[20] A. Quarteroni, A. Veneziani, Analysis of a geometrical multiscale model based on the coupling of ODE's and PDE's for blood flow simulations, Multiscale Model. Simul., Vol. 1, No. 2, 173-195, 2003.

[21] R. Temam, Navier-Stokes Equations, Theory and Numerical Analysis, North-Holland, Amsterdam, 1979.

[22] I.E. Vignon-Clementel, C.A. Figueroa, K.E. Jansen, C.A. Taylor, Outflow boundary conditions for three-dimensionnal finite element modelling of blood and pressure in arteries, Comput. Methods Appl. Mech. Engrg., 195, 3776-3796, 2006.

[23] R.G. Crystal, J.B. West, E.R. Weibel, P.J. Barnes, The lung : scientific foundations, Lippincott-Raven Press, Philadelphia, 1997. 